\title{
She's So Fine: Reflections on Whiteness, Femininity, Adolescence and Class in 1960s Music
}

Laurie Stras, editor

Farnham: Ashgate Publishing, 2011

ISBN 9781409436652 (PB)

RRP: £18.99 (GBP)

\author{
Nancy Bruseker \\ University of Liverpool \\ bruseker@liverpool.ac.uk
}

She's So Fine: Reflections on Whiteness, Femininity, Adolescence and Class in 1960 s Music is a collection of essays, loosely arranged in chronological order and covering American and British female singers of the 1960s. Included in this are Brenda Lee, Dusty Springfield, Tina Turner, Sandie Shaw, Mary Hopkin and Marianne Faithfull, along with collective discussion of many others. The scholarship collected in this volume is authored by some of the best known contributors to gender issues in popular music studies, which shows in the best possible way. At the outset, editor Laurie Stras asserts that this study works to make a unique contribution in scholarship, citing the devaluation of female voices in academic study as "footnotes to male-centred histories of popular culture and popular music, but [...] unless they are also songwriters they are equally disdained by most gender studies, even within musicology" (6). With a few notable examples - Madonna, the Spice Girls and certain jazz and blues icons - this is broadly an accurate observation.

The solid theoretical introduction and the close analysis of a number of well-known female performers does more with the material than a simple recasting of biographical detail. In addition to introducing the reader to well-known names, this collection seeks to speak to the sound of these women (or girls, in the historical taxonomy that ignores age). According to Stras, "most available writing on girl singers and girl groups is either historical or biographical journalism, or concentrates heavily on visual/textual analysis of image and lyric, bypassing musical-and especially the vocal-content" (23). Yet, the subtitle of the volume points to the need for contextual understanding to make sense of this content, as to varying degrees the chapters include analysis of the impact of age, class, race and gender, most doing so with a strong commitment to intersectionality.

The first of the essays, "Voice of the Beehive: Vocal Technique at the Turn of the 1960s", also written by Laurie Stras, offers the most in depth treatment of vocal technique. This article offers what is a useful approach popular music studies: a discussion of musical forms without recourse to musical notation. It is refreshing to read academic insight into the sound of the 1960s, and the women who helped produce it. Thus, Stras offers voice-models - imaginable, or available for listening on the internet - as categories of vocal technique. Here, technique is a neutral term to describe the method of producing sound, rather than exclusively suggesting training in 
bel canto technique (vocal training for formal/operatic singing). It allows analysis of non-classical vocal practice particular to popular music of the time; in the process, she demonstrates depth and nuance absent from other such discussion, specifically addressing Aida Pavletich's 'non technique'. The descriptions here serve not only to discuss the production of the unique sound of vocal music of the period, but also as an example for how vocal technique (in the broadest sense) should be discussed in other genres and time periods.

Similarly, Robynn Stilwell's analysis of Brenda Lee's voice, particularly in her childhood performance style, juxtaposes this with theories of childhood illuminated specifically through the aural. Here again, Stilwell uses non-notation methods to show the changes in performance style as Lee matured, allowing the non-musicologist to get to grips with the musical aspects of her performance, and its consequent implications. She also frames her reading of Lee's vocal technique through the lens of the 'blackness' of white trash in America: the peculiar crossover of class and race. Stilwell's view, that her voice is a key site for 'knowingness' is particularly compelling. Sarah Hill's contribution offers similar insights to Mary Hopkin's voice; without evaluation of its particular sound, the scholar is liable to misunderstand, or perhaps not understand at all, how she was heard by contemporary audiences. Jacqueline Warwick's "He hit me and I was glad" uses musical analysis to destabilize standard interpretations of the more problematic songs of the period, contextualizing it within the American civil rights movement and the social ramifications of African-American anger.

The essays in this collection make a point of discussing the musical aspect of female performance in the 1960s. While those discussed above are more explicit in their analysis, others take a lighter touch view, including Annie Randall's "Dusty's Hair", Norma Coates on Marianne Faithfull and Susan Fast on Tina Turner. It is the last of these essays which shows up the limitations of the volume. Fast rightly insists on the importance of scholars reading performance as well as listening to recordings, in order to properly understand Tina Turner's work in the 'Ike and Tina' years. Popular music studies is still a field in which the recording is king (gender intended).

In the introduction, Stras comments that, "[a]s we have sought to convey and discuss audiovisual experience without audiovisual aids, by concentrating on some of the better-known artists from the period, we hope our readers are able to summon up much of the material we consider in the mind's eye and ear" (23). While it is certainly possible to find and listen to examples of the work of the performers discussed herein by turning to the internet, a work such as this is hindered by the lack of such visual and auditory samples. Though the singers' often household names, not all readers will have a clear enough recollection of the described musical moments to ensure an appreciation of the analysis. This is particularly a generational issue. For example, Norma Coates's essay claims that [m]any young people grow up hearing and embracing, to an extent, their baby boomer parents' music." (184) However, this is a generation further back than the current generation of undergrads whose parents are Coates's 'young people'.

With the proliferation of online academic journals such as IASPM@Journal, and the ability for anyone to create wikis with embedded hyperlinks, the limitations of the paperformat volume could surely be obviated. This edited collection makes an important addition to such scholarship. Martha Mockus's response, at the end of the volume, concluded that '[b]ut there is more to do" (237), echoing Stras's call in the introduction: "[w]e would like to think of this volume as the beginning [...] there is so much more to say than a book of 100,000 -odd words can contain." (23) I wholeheartedly endorse this view, and thus highlight how much more engaging and compelling producing this work will be to the next generation of scholars, if only we can turn our attention to all the tools available to support it. 